\title{
Interface entre acreditação e segurança do paciente: perspectivas da equipe de enfermagem
}

\section{Interface between accreditation and patient safety: nursing team perspectives \\ Interfaz entre acreditación y seguridad del paciente: perspectivas del equipo de enfermería}

Como citar este artigo:

Oliveira JLC, Cervilheri AH, Haddad MCL, Magalhães AMM, Ribeiro MRR, Matsuda LM. Interface between accreditation and patient safety: nursing team perspectives. Rev Esc Enferm USP. 2020;54:e03604. DOI: https://doi.org/10.1590/S1980-220X2018053703604

João Lucas Campos de Oliveira ${ }^{1}$

Andressa Hirata Cervilheri ${ }^{2}$

Maria do Carmo Lourenço Haddad ${ }^{3}$

Ana Maria Müller de Magalhães ${ }^{1}$

Mara Regina Rosa Ribeiro ${ }^{4}$

Laura Misue Matsuda ${ }^{5}$

${ }^{1}$ Universidade Federal do Rio Grande do Sul, Escola de Enfermagem, Porto Alegre, RS, Brasil.

${ }^{2}$ Universidade Estadual de Maringá,

Programa de Pós-Graduação em

Enfermagem, Maringá, PR, Brasil.

${ }^{3}$ Universidade Estadual de Londrina,

Departamento de Enfermagem,

Londrina, PR, Brasil.

${ }^{4}$ Universidade Federal de Mato Grosso,

Faculdade de Enfermagem, Cuiabá, MT, Brasil.

${ }^{5}$ Universidade Estadual de Maringá,

Departamento de Enfermagem,

Maringá, PR, Brasil.

\section{ABSTRACT}

Objective: To understand the relationship between accreditation and patient safety from the perspective of the nursing team. Method: A descriptive-exploratory study implementing a qualitative approach. It was developed with nursing workers from two Intensive Care Units in a hospital certified in excellence by Brazilian accreditation. The participants responded to individual semi-structured interviews guided by the question: "Tell me about the relationship between accreditation and patient safety in this hospital and unit". The corpus was transcribed in full, and the thematic content analysis technique was used. Results: There were 14 professionals interviewed. There are several specific improvements in patient safety mediated by accreditation between the two emerging categories. The workers reported that at times the advances in safe care are transversally visible to the certification/maintenance of accreditation visit, and at times they point to safety as something independent of the quality seal. Conclusion: It was concluded that the investigated relationship was shown to be both dependent, as accreditation is a bridge for specific improvements, as well as independent, since patient safety goes beyond the certification process itself. In this context, criticality was revealed among nursing workers.

\section{DESCRIPTORS}

Hospital Accreditation; Patient Safety; Quality Management; Nursing, Team; Intensive Care Units.

Autor correspondente:

João Lucas Campos de Oliveira

Universidade Federal do Rio Grande

do Sul, Escola de Enfermagem

Rua São Manoel, 963, Santa Cecília

CEP 90620-110 - Porto Alegre, RS, Brasil

joao-lucascampos@hotmail.com 


\section{INTRODUÇÃO}

No setor saúde, a qualidade é um desafio ainda mais evidente aos gestores e profissionais que produzem os serviços, pois essa área de produção humana se distingue essencialmente dos demais setores de prestação de serviços pela característica peculiar de produção e consumo do "produto" - a assistência/o cuidado - concomitante entre $\mathrm{si}^{(1)}$.

Reconhecida a peculiaridade da qualidade na saúde, tem-se ainda que, defini-la propriamente é outro desafio, já que, uma vez legitimada pelos consumidores/usuários, principalmente, a conceituação do que se entende por cuidado qualificado tende a ser social e historicamente mutável ${ }^{(1-2)}$. Apesar disso, o uso otimizado de recursos disponíveis; a maior satisfação dos clientes internos e externos e; a redução de riscos associados ao cuidado, têm sido aceitos como a égide do entendimento de qualidade em saúde ${ }^{(2-3)}$.

Ao aceitar que o risco ou potencial danoso é inerente à produção assistencial, a segurança do paciente emerge como um dos pilares que fundamentam a própria qualidade, ou seja, segurança não é sinônimo de qualidade, mas é, sem dúvida, um dos seus pontos mais críticos/elementares ${ }^{(4)}$. Por este motivo, a Organização Mundial de Saúde elenca os seguintes preceitos básicos para o atendimento qualificado: eficácia, eficiência, acessibilidade, aceitabilidade, equidade e; mais recentemente, segurança ${ }^{(5)}$.

Apesar de ser um bem desejável que envolve a cultura organizacional sistêmica a seu favor, a segurança do paciente precisa ser viabilizada por práticas gerenciais concisas, proativas, compartilhadas, sistêmicas e sistematizadas, como: planejamento, educação permanente, gerenciamento de riscos, implantação de protocolos assistenciais e principalmente, avaliação cíclica das estratégias implantadas, seguida de (re) planejamentos ${ }^{(6)}$.

No contexto da avaliação em saúde, a acreditação tem despontado em todo o mundo como um sistema de gestão calcado essencialmente em atividades avaliativas externas às organizações aderentes, sendo muito bem adaptado à realidade singular da área da saúde ${ }^{(7-8)}$.

A essência da acreditação entorna-se pela comparação da realidade institucional, avaliada externa e periodicamente à luz de padrões de qualidade previamente definidos, os quais são determinados de forma clara e normatizada pela metodologia acreditadora aderida por cada país que, no caso do Brasil, é representada pela Organização Nacional de Acreditação (ONA), além de outras advindas de realidades norte-americanas como a Joint Comission Internacional, Canadian Accreditation Council, e National Integrated Accreditation for Healthcare Organizations ${ }^{(7)}$. A certificação é um produto muito bem-vindo da acreditação, no entanto, em tese, não é a sua finalidade prioritária, mas sim a melhoria contínua, a educação permanente sistêmica para a cultura de qualidade e o uso de ferramentas sistemáticas ${ }^{(9)}$

A acreditação é um sistema de gestão da qualidade que tende a ser conduzido por meio do estabelecimento de critérios, padrões e indicadores, ou seja, alavanca a visão estratégica das instituições, incluindo aspectos de marketing e incremento gerencial em diversas esferas organizacionais ${ }^{(8,10-11)}$.
$\mathrm{Na}$ metodologia de acreditação mediada pela ONA, os critérios do primeiro (de três) nível de certificação correspondem, essencialmente, às práticas que são voltadas à segurança do paciente ${ }^{(12)}$.

Apesar da relação "evidente" entre acreditação e segurança do paciente, uma vez que a segunda é um dos focos centrais do sistema de gestão da qualidade em pauta, estudos que viabilizem o melhor/maior conhecimento sobre os efeitos da acreditação em diferentes aspectos têm sido recomendados por pesquisas publicadas recentemente, não somente no Brasil ${ }^{(7-8,10,13-15)}$, ou seja, investigar os potenciais deste processo de certificação da qualidade - o que sem dúvida inclui a segurança do paciente - ainda denota ineditismo e, principalmente, necessidade. Ademais, mesmo que exista este gap no conhecimento, parte-se do princípio empírico de que existe alguma relação, seja ela positiva ou não, entre os fenômenos elencados.

Considerando que a equipe de enfermagem é protagonista nas ações que favorecem a (in)segurança do paciente devido a sua participação fundamental e ininterrupta nos processos de cuidado, em especial no ambiente hospitalar, emerge a importância de investigar a problemática antes enunciada com enfoque nesta população. Sendo assim, este estudo se pauta na questão: Quais são as perspectivas da equipe de enfermagem sobre a relação entre acreditação e segurança do paciente? Portanto, o objetivo consistiu em apreender a relação entre acreditação e segurança do paciente, na perspectiva da equipe de enfermagem.

\section{MÉTODO}

\section{Desenho do estudo}

Estudo descritivo-exploratório de natureza qualitativa.

\section{CenÁrio}

O local de pesquisa foi um hospital geral situado no estado do Paraná, Brasil, detentor do certificado de acreditação.

\section{CRITÉRIOS DE SELEÇÃo}

A seleção do hospital se deu por busca no sítio eletrônico online da ONA por um hospital geral com leitos de terapia intensiva, selado pelo maior nível de certificação da ONA (Acreditado com Excelência), com maior tempo de validade do selo de qualidade, e situado no estado do Paraná, Brasil.

A definição do critério de certificação em excelência foi intencional, ao entendimento de que os trabalhadores neste tipo de organização poderiam ter maior conhecimento sobre a relação entre acreditação e segurança do paciente. A presença de Unidade de Terapia Intensiva (UTI) na organização foi prevista ao claro conhecimento de que esses setores impõem alta periculosidade à segurança do paciente. Já a delimitação geográfica ao estado do Paraná deu-se em virtude de aumentar a viabilidade do estudo, sediado no mesmo estado.

A população da pesquisa compreendeu profissionais de enfermagem lotados nas duas UTI para adultos do hospital acreditado, sendo uma delas de atendimento geral e outra 
especializada em assistência coronariana. A amostra $(n=14)$ foi composta através da abordagem por conveniência, pelos trabalhadores que correspondessem ao único critério de elegibilidade, independente se enfermeiro ou profissional de nível médio, a saber: atuar na unidade há pelo menos seis meses. Este critério foi definido a fim de que o trabalhador tivesse condições/subsídios de responder ao propósito do estudo.

\section{Coleta de Dados}

A coleta de dados foi procedida em junho de 2017 por aplicação de formulário para caracterização sociodemográfica e laboral dos participantes, e entrevista semiestruturada, gravada à anuência dos trabalhadores de enfermagem, norteada pela seguinte questão: "Fale-me sobre a relação entre acreditação e segurança do paciente neste hospital e unidade”. O número de entrevistas foi definido quando os depoimentos se tornaram sucessivamente repetitivos, ou seja, houve saturação dos dados. Todos os dados extraídos dos depoimentos foram integralmente transcritos em meio digital, e, posteriormente, impressos.

\section{ANÁLISE E TRATAMENTO DOS DADOS}

Ao corpus empírico dos dados, em meio impresso, foi procedida a Análise de Conteúdo, na modalidade Temática, respeitando-se as etapas de pré-análise, exploração do material e interpretação dos dados ${ }^{(16)}$.

A pré-análise compreendeu a identificação das ideias centrais dos depoimentos, que significam os pontos-chave de suas falas, devidamente destacadas pelo pesquisador por meio da leitura flutuante (primária) do material empírico ${ }^{(16)}$. Já a exploração do material empírico consistiu de repetidas leituras sobre o corpus, procedimento que evidenciou as ideias centrais anteriormente destacadas e deu luz aos núcleos de sentido, ou seja, cernes de significados emitidos pelos depoentes, que são oriundos da leitura aprofundada do conteúdo emitido pelos depoentes ${ }^{(16)}$.

Por fim, através da análise dos núcleos de sentido, os dados foram (re)interpretados, dando teor sistemático às categorias, advindas das etapas anteriores e que significam a condensação nominal do conteúdo semântico emitido pelos participantes $^{(16)}$.

$\mathrm{Na}$ apresentação dos resultados, os excertos/trechos/verbatins dos depoimentos extraídos das entrevistas analisadas foram editados à norma culta, sem, no entanto, alterar o sentido da emissão. Termos entre colchetes foram acrescentados quando na necessidade de melhor compreensão do depoimento pelo leitor. Os entrevistados foram identificados pela letra "E" seguida de um número arábico de 1 a 14, de acordo com a ordem cronológica das entrevistas.

\section{Aspectos étICOS}

Todos os princípios éticos da Resolução n. 466/12, do Conselho Nacional de Saúde, que regem as pesquisas com seres humanos foram integralmente cumpridos, o que inclui a devida distribuição, leitura e assinatura - em duas vias de igual teor - do Termo de Consentimento Livre e Esclarecido por todos os participantes. O Projeto de Pesquisa que fomentou este desdobramento de estudo foi submetido e apreciado por Comitê de Ética Institucionalizado mediante Parecer no 1.788.249/2016.

\section{RESULTADOS}

O hospital selecionado se caracterizava como de médio porte, com leitos disponíveis ao Sistema Único de Saúde, localizado no interior do Paraná, com certificação de acreditação em nível de excelência ("Nível 3" - ONA) desde 2014.

Dos 14 profissionais entrevistados, apenas um era homem. Doze profissionais eram casados, e dois, solteiros. A distribuição entre enfermeiros e técnicos de enfermagem foi igualitária (sete em cada categoria profissional). A média de idade dos trabalhadores foi de 36 anos $( \pm 8,6)$.

À análise temática do conteúdo das entrevistas, emergiram duas categorias: (1) Melhorias pontuais na segurança do paciente advindas da acreditação hospitalar; e, (2) Segurança do paciente: um bem (in)dependente da certificação de acreditação?

\section{MelHorias PONTUAIS NA SEGURANÇA do PACIENTE ADVINDAS DA ACREDITAÇÃO HOSPITALAR}

Os trabalhadores de enfermagem reconheceram a acreditação como meio de impulsionar/incrementar o cuidado seguro, em especial, pelo uso racional de protocolos e estratégias de segurança assistencial, os quais foram demandados à acreditação:

(...) desde que o paciente chega, ele receba a identificação, tanto a pulseira quanto a placa de identificação no leito. As fitas coloridas, que cada uma diz respeito a uma coisa. O risco de queda do paciente (...) A gente teve troca de camas recentemente. Todas as camas estão com grades boas, são camas elétricas (E6).

Antigamente, a gente não identificava o paciente. Hoje, as medicações de alto risco já vêm identificadas, vem com uma etiqueta bem grande, em vermelho, para ver que é perigoso (...) As pulseirinhas de protocolo: dor torácica, pessoas que têm fístula, têm as de lesão por pressão (...) (E8).

Acho que tem tudo a ver. A acreditação, ela vem para humanizar esse cuidado, o tratamento, e a segurança do paciente. Eu vejo que a acreditação vem para focar, realmente, no paciente, na segurança dele. Para controlar infecção e essas coisas (...) (E10).

Nomina-se claramente diversos protocolos e/ou meios de segurança viabilizados (exigidos) pela acreditação, reforçando o sistema de gestão em pauta como alicerce às melhorias pontuais no cuidado seguro:

A identificação do paciente é uma exigência da acreditação. Ela previne vários eventos adversos, e a prática de você nunca chegar a um paciente e aplicar uma medicação sem antes fazer a dupla checagem (...) A parte da cirurgia segura, é um protocolo também para a segurança do paciente, que está seguindo todos os passos da cirurgia segura (...) Prevenção de PAV [pneumonia associada à ventilação], que é manter o leito elevado à $45^{\circ}$, realizar aspiração. Temos cuidados que a gente tem que seguir para evitar a $P A V(. .$.$) A acreditação fomenta isso (...) A acreditação$ é isso. Eles veem, de certa forma, como uma fiscalização, mas é 
com intenção de sempre estar dando dicas para que o hospital melhore (E12).

Adiante, também elencam algumas melhorias na segurança do paciente conectadas à acreditação do hospital empregador:

Nós observamos algumas mudanças, principalmente em relação à medicação. Foram muitas mudanças que ajudaram, criaram barreiras [de segurança], e até mesmo na forma de trabalhar com opaciente (E4).

(...) Protocolo de sepse. Entrou no protocolo de sepse, você tem que coletar todos os exames de rotina e iniciar o antibiótico (E7).

Todos os protocolos que funcionam criam interações entre os processos. Acho bem legal, e a gente procura seguir. E tem os eventos [adversos]. Baseado nos eventos tem as falhas, procura-se ver as interações, as não conformidades (...) Ela [acreditação] visa à melhoria do paciente (E14).

Por fim, demonstra-se outros excertos que legitimam a relação entre a acreditação e a segurança do paciente, na perspectiva da equipe de enfermagem.

Trouxe mudanças. A acreditação traz um gerenciamento $e$ supervisão. Então, se tem alguém que supervisione, que cobre aqueles protocolos [de segurança], eles vão funcionar (E13).

Querendo ou não, a segurança do paciente envolve tudo: pessoal da recepção, da limpeza, do administrativo. Tudo vai envolver a segurança do paciente. Eu acho que, com o fato de vir a acreditação, pelo menos a gente tem um norte, tem passos a serem seguidos. Direciona aquilo que a gente tem que fazer para o paciente ter, realmente, segurança (E6).

Apesar da clara menção, até mesmo pontual, de melhorias na segurança do paciente possibilitadas pela acreditação hospitalar, os trabalhadores também emitiram conteúdo que condensou a categoria seguinte. Esta, se trata da segurança do paciente por ora vista como fator dependente da visita de certificação para acreditação, outrora, vista como independente do selo de qualidade, dualidade esta que rotulou interrogativa à temática.

\section{SeguranÇa do Paciente: um bem (IN)Dependente DA CERTIFICAÇ̃̃O DE ACREDITAÇÃO?}

Os trabalhadores de enfermagem denotam que a segurança do paciente é mais visível no hospital nos momentos pontuais que perpassam a visita de avaliação e/ou certificação de acreditação:

(...) porque muitas vezes a gente vê tantos erros que acontecem, e você vai ver quando está se aproximando da certificação, as pessoas começam a melhorar para eles [auditores externos] não pegarem [notarem] (...) (E1).

Na teoria é tudo muito lindo. Na prática, eu ainda acho que tem que melhorar. Por exemplo, quando a acreditação está aqui: maravilha. Acho que tem que existir um meio de que isso seja colocado na prática (E4).

Eu acho que quando está passando o pessoal da acreditação aqui, para fazer [a avaliação] é aquele alvoroço, e depois, parece que murcha. Não falo assim, de não fazer as coisas, mas, às vezes é tanta cobrança e depois assim [desanda] (...) Entendeu? (E9).

Outro profissional de enfermagem relaciona claramente a avaliação externa como fator dependente às melhorias na segurança do paciente:

Então, o pessoal do [nome da Instituição Acreditadora] vem e verifica o fluxo de trabalho, se os protocolos estão sendo seguidos, e colocam metas. Em relação à segurança do paciente, eles querem exigir excelência no atendimento. Claro que nunca vai ser $100 \%$, mas eu acredito que isso, essa verificação, faz todo mundo se envolver (...) (E12).

Ainda que dual, de forma igualmente crítica, os trabalhadores de enfermagem do hospital acreditado mencionam que a segurança do paciente não deve depender da certificação de acreditação:

(...) acreditação eu vejo que é um conjunto de, não digo de normas, mas de critérios para se alcançar um título, um status, um certificado. E, a segurança do paciente vai muito além da acreditação (...) Então, para nós, a segurança do paciente é o primeiro critério a seguir (E13).

(...) mas eu acho que não precisaria da acreditação para estar fazendo isso [melhorias na segurança do paciente]. O conhecimento acadêmico dos profissionais (...) poderia estar fazendo tudo isso sem a intervenção da acreditação (E7).

A segurança do paciente tem que ter independente de qualquer coisa. Acho que contribui, mas não altera. Acho que se o hospital não fosse acreditado, a segurança seria igual (E11).

\section{DISCUSSÃO}

As estratégias de segurança assistencial implementadas na instituição advindas do processo de acreditação coadunam com os critérios avaliados pela ONA, uma vez que, para a certificação desde o primeiro nível, são exigidos requisitos básicos da qualidade na assistência prestada voltados para o princípio de segurança em todas as áreas de atividade ${ }^{(12)}$. Logo, a primeira categoria temática, de certa forma, legitima que os padrões de segurança exigidos são reconhecidos na perspectiva da equipe de enfermagem, o que é positivo.

No processo de acreditação, é comum o estabelecimento de ações sistemáticas para padronização por meio da implantação de protocolos voltados à procedimentos assistenciais $^{(8)}$, conforme mencionado nos excertos de E6, E8 e E10, que discorrem sobre processos direcionados de acordo com os protocolos de segurança. Isso é importante, inclusive externamente ao contexto da acreditação, pois, a adoção de boas práticas determinadas por protocolos é uma recomendação ao alcance e incremento da segurança nos processos assistenciais $^{(17)}$.

Cabe considerar que a acreditação favorece a cultura de segurança na instituição evidenciada diante da menção dos protocolos de segurança relacionados aos processos e melhorias, conforme fragmentos dos relatos contidos no primeiro eixo, tais como o uso de pulseira e placa de identificação, prevenção de quedas por meio do uso de grades em todas as camas, controle de medicações de alto risco e controle de 
infecção hospitalar. Tais ações são fundamentais à melhoria da qualidade hospitalar e segurança do paciente internado, e, não somente a sua implantação é elementar, como também, o monitoramento através de medidas cíclicas e sistematizadas de avaliação $^{(4)}$.

Outros aspectos relacionados a segurança do paciente alicerçados à certificação de acreditação foram mencionados, entre eles, o monitoramento da instituição acreditadora como estímulo às melhorias no hospital, conforme fala de $\mathrm{E} 12$, as barreiras de segurança citadas por E4 e as interações entre os processos que permeiam os serviços prestados na instituição e que provieram dos protocolos de segurança, mencionadas por E14. Todo este arsenal foi elencado como subsídio às melhorias no cuidado seguro, concordando com a eficácia da acreditação na melhoria da assistência e segurança do paciente ${ }^{(18)}$, o que, possivelmente, se relaciona à uma cultura de qualidade e segurança mais bem desenvolvida na organização acreditada.

Problematiza-se, portanto, que os profissionais demonstram ter incorporado novas formas de atuar em relação aos eventos do cotidiano que interferem na segurança do paciente. Isso é louvável e, até mesmo, uma premissa básica da acreditação, pois seus métodos bem definidos buscam reforçar a racionalidade do trabalho em saúde a fim de subsidiar a qualidade estratégica e a promoção da cultura de melhoria da mesma ${ }^{(8,12)}$.

Considerando que a cultura em uma organização é instituída diante dos valores, comportamentos e práticas compartilhadas entre as pessoas que estão inseridas no processo de trabalho ${ }^{(19)}$, evidencia-se a cultura de segurança estabelecida na instituição uma vez que os verbatins da primeira categoria temática referenciam protocolos e estratégias de segurança assistencial advindos da acreditação. Porém, é prudente interpretar criticamente esse dado, já que a implantação de ações sistêmicas e racionais não exclui o comportamento proativo (inclusive das chefias) à melhoria contínua e o aprendizado com os erros, que também são premissas básicas de uma cultura organizacional positiva para o cuidado seguro ${ }^{(20)}$.

A respeito da melhoria contínua, um estudo recente desenvolvido em Abu Dhabi, por meio de análise em séries temporais ao longo de oito anos em diferentes ciclos de acreditação, demonstrou que o sistema de gestão da qualidade tende a sustentar melhorias na qualidade do cuidado direto, isso porque os autores inferem que, ao se submeter à primeira visita de certificação, os melhores desempenhos tendem a se sustentar ao longo do tempo, solidificando maior confiabilidade ao processo de melhoria ${ }^{(21)}$.

Pondera-se sobre os resultados assistenciais efetivos diante da cultura de segurança relacionada à acreditação, uma vez que há correlação significativa entre cultura de segurança e resultados de segurança hospitalar ${ }^{(22)}$, bem como associação consistentemente positiva entre cultura de segurança e resultados de pacientes relacionados ao sistema, tais como taxas de mortalidade, falha no resgate, taxas de readmissão e eventos adversos/erros de medicação ${ }^{(19)}$.

Ao fomentar a cultura de melhoria no cuidado seguro, neste estudo, traduzida principalmente pela incorporação de ações racionais de segurança do paciente, o hospital acreditado parece ir ao encontro da cultura de segurança positiva. Todavia, destaca-se que a cultura de segurança, por si só, pode e deve ser mensurada ${ }^{(20,22)}$, o que já evidencia caminho(s) de pesquisa(s) futura(s).

$\mathrm{Na}$ perspectiva da enfermagem, houve reconhecimento da relação entre a acreditação e a segurança do paciente, conforme excerto de E13 ao mencionar o direcionamento que o processo de certificação promove por meio da supervisão das ações, pois tal "cobrança" é vista como uma garantia do cumprimento aos protocolos. Ainda, o norteamento promovido pela acreditação como forma eficaz na garantia de segurança do paciente também foi elencado por E6, o qual complementa a afirmativa da presença da cultura de segurança ao mencionar que a segurança permeia todos as áreas da instituição, incluindo as áreas de apoio, como o setor de higiene, até as áreas administrativas, fato que coaduna aos princípios de avaliação sistêmica da $\mathrm{ONA}^{(12)}$.

Os resultados do estudo e da literatura apresentam uma convergência no sentido de que a acreditação pode contribuir para o fortalecimento de uma cultura institucional de segurança dos processos assistenciais, tendo o paciente como o centro do cuidado, envolvendo todas as equipes de forma transversal, promovendo a padronização de procedimentos e auxiliando no fomento de uma cultura de aprendizagem com as falhas que acontecem nos processos.

Apesar da acreditação ter sido elencada como meio de favorecimento a ações de segurança do paciente, os trabalhadores mencionaram aspectos que geram dualidade na relação de dependência entre a acreditação e segurança do paciente. Por um lado, a segurança do paciente é mais evidente nos momentos prévios ou pontuais da visita de avaliação e/ou certificação, conforme excertos de E1, E4 e E9, ao mencionarem que há mobilização para que as melhorias sejam colocadas em prática principalmente quando a instituição avaliadora se faz presente.

$\mathrm{O}$ fato antes descrito pode ser discorrido na perspectiva do monitoramento previsto das acreditação, que promove na instituição um estímulo para a realização das ações exigidas para a conquista/manutenção da certificação ${ }^{(12)}$. Tal monitoramento é realizado tanto por auditorias internas que ocorrem no hospital, como também, nas visitas de manutenção já previstas à acreditação, sendo consideradas como ferramentas importantes na avaliação da qualidade ${ }^{(12,23-24)}$.

O monitoramento da qualidade assistencial é uma prática de gestão estratégica e racional que independe da certificação de acreditação ${ }^{(4,9)}$. Tendo isso em pauta, volta-se ao fato constatado de que as melhorias ou adesão às ações de segurança são mais evidentes quando no momento transversal à visita de certificação/manutenção de forma bastante crítica, pois isso, inevitavelmente, gera um teor de fiscalização à acreditação. Destarte, a fiscalização contraria os princípios elementares de melhoria proativa da qualidade da acreditação, mesmo que estes sejam balizados pela avaliação externa e a rigorosidade no cumprimento de padrões ${ }^{(12)}$.

Reforça-se que denotar melhor/maior adesão a práticas de segurança do paciente de forma dependente à visita de certificação/manutenção da acreditação pode ser essencialmente prematuro porque isso, de certa forma, pode levar a 
ideia de que, fora do contexto de avaliação externa, o hospital não evidencia (ao menos tão incisivamente) sua preocupação com o cuidado seguro, que é direito dos usuários independentemente da inserção da instituição em um serviço certificado pela acreditação. Por outro lado, a segurança do paciente foi elencada como fator independente da certificação de acreditação, o que é muito positivo.

Os trabalhadores consideraram a segurança do paciente como primordial na prática assistencial, que não deve estar ligada como produto da certificação, mas sim independente e além desta, evidenciado nos excertos de E13, E7 e E11. Tal assertiva pode estar embasada na valorização da segurança do paciente na perspectiva dos profissionais de enfermagem, sendo fundamental na consolidação de uma cultura de segurança construtiva ${ }^{(25)}$.

Postula-se que posicionar a segurança do paciente como produto independente da certificação de acreditação não é somente positivo, como também ético e muito crítico da parte dos trabalhadores de enfermagem. Isso porque, é eticamente prudente reconhecer que o cuidado seguro transpõe a dinâmica administrativo-estratégica comum dos sistemas de gestão da qualidade hospitalar, como a acreditação ${ }^{(8)}$, indo além disso, mesmo que tais sistemas, inclusive conforme constatado neste estudo, alavanquem a robustez das ações de segurança do paciente.

O cenário contraditório aqui constatado reforça o ineditismo e relevância deste estudo, qual seja: na perspectiva da equipe de enfermagem, as ações de segurança do paciente são ampliadas e a mobilização de profissionais também, quando na proximidade da visita de avaliação de acreditação, depois se amenizam. Em contraponto, os mesmos trabalhadores inferem ora que as ações concretas de segurança do paciente são dependentes ou propriamente produtos da acreditação, ora que a segurança é um bem muito maior do que o selo de qualidade, fato aqui interpretado como essencialmente positivo.
É evidente que este estudo não esgota o conhecimento da relação/interface entre a acreditação e a segurança do paciente. Ademais, são algumas limitações da pesquisa: a impossibilidade de generalização dos resultados (apesar de isso não ser previsto ao desenho do estudo), bem como a inclusão de uma única categoria profissional.

Apesar do exposto anterior, acredita-se que a menção de estratégias pontuais de segurança advindas da acreditação e também o fato da extensa criticidade dos trabalhadores de enfermagem ao posicionarem a segurança do paciente como bem "superior" ao selo de qualidade são contribuições importantíssimas à área de gestão da qualidade e segurança do paciente. Todavia, a impossibilidade de afirmar objetivamente melhores resultados de segurança do paciente no hospital acreditado é uma grande perspectiva para estudos futuros, incluindo os de abordagem quantitativa e/ou mista.

\section{CONCLUSÃO}

Conclui-se que a perspectiva da equipe de enfermagem sobre a relação entre acreditação e segurança do paciente se refere à pontuação clara de meios/estratégias de cuidado mais seguro advindos do processo de acreditação, em especial por aspectos de teor instrumental e sistemático embutidos no processo de traba1ho. Pontuam, ainda, a segurança do paciente ora como dependente (por meio dos momentos transversais de manutenção do selo de qualidade), ora como independente da acreditação, já que o cuidado seguro transpõe (é "maior") que a certificação em si. Nesse contexto, revelou-se valorização do processo de acreditação rumo à assistência mais segura, mas, também, criticidade entre os trabalhadores de enfermagem.

\section{RESUMO}

Objetivo: Apreender a relação entre acreditação e segurança do paciente, na perspectiva da equipe de enfermagem. Método: Estudo descritivo-exploratório, de natureza qualitativa. Foi desenvolvido com trabalhadores de enfermagem de duas Unidades de Terapia Intensiva de um hospital certificado em excelência pela acreditação brasileira. Os participantes responderam a entrevistas individuais semiestruturadas, norteadas pela questão: "Fale-me sobre a relação entre acreditação e segurança do paciente neste hospital e unidade". Ao corpus transcrito na íntegra, empregou-se análise de conteúdo temática. Resultados: Foram entrevistados 14 profissionais. Entre as duas categorias que emergiram, relaciona-se diversas melhorias pontuais na segurança do paciente mediadas pela acreditação. Os trabalhadores referem que: ora os avanços no cuidado seguro são visíveis transversalmente à visita de certificação/manutenção da acreditação, ora pontuam a segurança como algo independente do selo de qualidade. Conclusão: Concluiu-se que a relação investigada tanto se mostrou como dependente, pois a acreditação é ponte para melhorias pontuais, como independente, já que a segurança do paciente transpõe o processo de certificação em si. Nesse contexto, revelou-se criticidade entre os trabalhadores de enfermagem.

\section{DESCRITORES}

Acreditação Hospitalar; Segurança do Paciente; Gestão da Qualidade; Equipe de Enfermagem; Unidades de Terapia Intensiva.

\section{RESUMEN}

Objetivo: Aprender la relación entre acreditación y seguridad del paciente, en la perspectiva del equipo de enfermería. Método: Estudio descriptivo-exploratorio, de naturaleza cualitativa. Fue desarrollado con trabajadores de enfermería de dos Unidades de Terapia Intensiva de uno hospital certificado en excelencia por la acreditación brasileña. Los participantes responderán a entrevistas individuales semiestructuradas, guiadas por la cuestión: "Habla-me acerca de la relación entre acreditación y seguridad del paciente en esto hospital y unidad". $\mathrm{Al}$ corpus transcrito en su integridad, utilizó el análisis del contenido temático. Resultados: Fueron entrevistados 14 profesionales. Entre las dos categorías que emergieran, relaciona se diversas mejoras puntuales en la seguridad de lo paciente mediadas por la acreditación. Los trabajadores refieren que: ora los avanzos en el cuidado seguro es visible transversalmente a la visita de la certificación/manutención de la acreditación, ora puntúan la seguridad como algo independiente del sello de calidad. Conclusión: Se concluye, pues la acreditación es puente para mejoras puntuales, como independiente, ya que la seguridad de lo paciente transpone el proceso de certificación en sí mismo. En esto contexto, revela se criticidad entre los trabajadores de enfermería.

\section{DESCRIPTORES}

Acreditación de Hospitales; Seguridad del Paciente; Gestión de la Calidad; Grupo de Enfermería; Unidades de Cuidados Intensivos. 


\section{REFERÊNCIAS}

1. Oliveira JLC, Matsuda LM. Acreditação: possibilidade para o avanço da gestão da qualidade em saúde e enfermagem? Ciênc Cuid Saúde. 2015;14(2):993-4. DOI: 10.4025/cienccuidsaude.v14i2.28142

2. Vituri DW, Évora YDM. Total Quality Management and hospital nursing: an integrative literature review. Rev Bras Enferm. 2015;68(5):660-7. DOI: http://dx.doi.org/10.1590/0034-7167.2015680525i

3. Donabedian A. The seven pillars of quality. Arch Pathol Lab Med. 1990;114(11):1115-8.

4. Tres DP, Oliveira JLC, Vituri DW, Alves SR, Rigo DFH, Nicola AL. Quality of care patient safety: assessment based on indicators. Cogitare Enferm. 2016;21(n.esp.):1-7. DOI: http://dx.doi.org/10.5380/ce.v21i5.44938

5. World Health Organization. Quality of Care: a process for making strategic choices in systems [Internet]. Geneva: WHO; 2006 [cited 2018 Sep 05]. Available from: https://www.who.int/management/quality/assurance/QualityCare_B.Def.pdf

6. Reis GAX, Valera IMA, Inoue KC, Souza VS, Costa MAR, Matsuda LM. Implantação das estratégias de segurança do paciente: sugestões de enfermeiros gestores. Vigil Sanit Debate. 2016;4(4):132-8. DOI: 10.22239/2317-269X.00801

7. Berssaneti FT, Saut AM, Barakat MF, Calarge FA. Is there any link between accreditation and models of organizational excellence? Rev Esc Enferm USP. 2016;50(4):650-7. DOI: http://dx.doi.org/10.1590/S0080-62342016000050016

8. Oliveira JLC, Gabriel CS, Fertonani HP, Matsuda LM. Management changes resulting from hospital accreditation. Rev Latino Am Enfermagem. 2017;25:e2851. DOI: http://dx.doi.org/10.1590/1518-8345.1394.2851

9. Oliveira JLC, Matsuda LM. Disqualification of certification by hospital accreditation: Perceptions of professional staff. Texto Contexto Enferm. 2016; 25(1):e4430014. DOI: http://dx.doi.org/10.1590/0104-07072016004430014

10. Caldana G, Gabriel CS. Evaluation of the hospital accreditation program: face and content validation. Rev Bras Enferm. 2017;70(1):47-53. DOI: http://dx.doi.org/10.1590/0034-7167-2016-0184

11. Gabriel CS, Bogarin DF, Mikael S, Cummings G, Bernardes A, Gutierrez L, et al. Perspectiva de las enfermeras brasileñas sobre el impacto de la Acreditación Hospitalaria. Enferm Glob. 2017;17(49):381-94 DOI: https://doi.org/10.6018/eglobal.17.1.283981

12. Organização Nacional de Acreditação. Manual das organizações prestadoras de serviços de saúde. Brasília: ONA; 2018.

13. Bogh SB, Falstie-Jensen AM, Bartels P, Hollnagel E, Johnsen SP. Accreditation and improvement in process quality of care: a nationwide study. Int J Qual Health Care. 2015;27(5):336-43. DOI: 10.1093/intqhc/mzv053

14. Bogh SB, Falstie-Jensen AM, Hollnagel E, Holst R, Braithwaite J, Johnsen SP. Improvement in quality of hospital care during accreditation: A nationwide stepped-wedge study. Int J Qual Health Care. 2016;28(6):715-20. DOI: 10.1093/intqhc/mzw099

15. Algahtani H, Aldarmahi A, Manlangit Jr J, Shirah B. Perception of hospital accreditation among health professionals in Saudi Arabia. Ann Saudi Med. 2017;37(4):326-32. DOI: 10.5144/0256-4947.2017.326

16. Bardin L. Análise de conteúdo. Lisboa: Edições 70; 2011.

17. Paixão DPSS, Batista J, Maziero ECS, Alpendre FT, Amaya MR, Cruz EDA. Adhesion to patient safety protocols in emergency care units. Rev Bras Enferm. 2018;71(Suppl 1):577-84. DOI: http://dx.doi.org/10.1590/0034-7167-2017-0504

18. Reisi N, Raeissi P, Sokhanvar M, Kakemam E. The impact of accreditation on nurses' perceptions of quality of care in Iran and its barriers and facilitators. Int J Health Plann Manage. 2019;34:e230-40. DOI: 10.1002/hpm.2642

19. Braithwaite J, Herkes J, Ludlow K, Testa L, Lamprell G. Association between organisational and workplace cultures, and patient outcomes: systematic review. BMJ Open. 2017;7:e017708. DOI: 10.1136/bmjopen-2017-017708

20. Costa DB, Ramos D, Gabriel CS, Bernardes A. Cultura de segurança do paciente: avaliação pelos profissionais de enfermagem. Texto Contexto Enferm. 2018;27(3):e2670016. DOI: http://dx.doi.org/10.1590/0104-070720180002670016

21. Devkaran S, O'Farrell PN, Ellahham S, Arcangel R. Impact of repeated hospital accreditation surveys on quality and reliability, an 8-year interrupted time series analysis. BMJ Open. 2019;15;9(2):e024514. DOI: http://dx.doi.org/10.1136/bmjopen-2018-024514

22. Saleh AM, Darawad MW, Al-Hussami M. The perception of hospital safety culture selected outcomes among nurses: an exploratory study. Nurs Health Sci. 2015;17:339-46. DOI: 10.1111/nhs.12196

23. Andrade LZ, Prado MA, Barbosa MA, Silva LCS, Gebrim CFL, Brasileiro ME, et al. Internal audit as a facilitator instrument for hospital accreditation. PIJR. 2017;6(9):625-9.

24. Hanskamp-Sebregts M, Zegers M, Westert GP, Boeijen W, Teerenstra S, Van Gurp PJ, et al. Effects of patient safety auditing in hospital care: results of a mixed-method evaluation (part 1). Int J Qual Health Care. 2019;31(7):G8-15 DOI: https://doi.org/10.1093/intqhc/mzy134

25. Silva-Batalha EMS, Melleiro MM. Cultura de segurança do paciente: percepções da equipedeenfermagem. HU Rev (Juiz de Fora) [Internet]. 2016 [citado 2018 nov. 20];42(2):133-42. Disponível em: https://hurevista.ufjf.emnuvens.com.br/hurevista/article/view/2518 